\title{
Spatially Specific Working Memory Activity in the Human Superior Colliculus
}

\author{
Masih Rahmati, ${ }^{1,2}$ Kevin DeSimone, ${ }^{1,2}$ Clayton E. Curtis, ${ }^{1,3}$ and Kartik K. Sreenivasan ${ }^{2}$ \\ ${ }^{1}$ Department of Psychology, New York University, New York, New York 10003, ${ }^{2}$ Division of Science and Mathematics, New York University Abu \\ Dhabi, Abu Dhabi, UAE, and ${ }^{3}$ Center for Neural Science, New York University, New York, New York 10003
}

Theoretically, working memory (WM) representations are encoded by population activity of neurons with distributed tuning across the stored feature. Here, we leverage computational neuroimaging approaches to map the topographic organization of human superior colliculus (SC) and model how population activity in SC encodes WM representations. We first modeled receptive field properties of voxels in SC, deriving a detailed topographic organization resembling that of the primate SC. Neural activity within human ( 5 male and 1 female) SC persisted throughout a retention interval of several types of modified memory-guided saccade tasks. Assuming an underlying neural architecture of the SC based on its retinotopic organization, we used an encoding model to show that the pattern of activity in human SC represents locations stored in WM. Our tasks and models allowed us to dissociate the locations of visual targets and the motor metrics of memory-guided saccades from the spatial locations stored in WM, thus confirming that human SC represents true WM information. These data have several important implications. They add the SC to a growing number of cortical and subcortical brain areas that form distributed networks supporting WM functions. Moreover, they specify a clear neural mechanism by which topographically organized SC encodes WM representations.

Key words: fMRI; human; modeling; retinotopy; saccades; working memory

\section{Significance Statement}

Using computational neuroimaging approaches, we mapped the topographic organization of human superior colliculus (SC) and modeled how population activity in SC encodes working memory (WM) representations, rather than simpler visual or motor properties that have been traditionally associated with the laminar maps in the primate SC. Together, these data both position the human SC into a distributed network of brain areas supporting WM and elucidate the neural mechanisms by which the SC supports WM.

\section{Introduction}

We have known for decades that working memory (WM) relies on the persistent activity of neurons selective for memoranda (Funahashi et al., 1989; Miller et al., 1996). Theoretical work suggests that WM features are encoded by the joint activity of ensembles of neurons with tunings that span the memorized

Received Aug. 1, 2020; revised Sep. 26, 2020; accepted 0ct. 21, 2020.

Author contributions: M.R. and K.D. analyzed data; M.R., K.D., C.E.C., and K.K.S. wrote the first draft of the paper; M.R., C.E.C., and K.K.S. edited the paper; M.R., C.E.C., and K.K.S. wrote the paper; K.D., C.E.C., and K.K.S. designed research; K.D. performed research.

The authors declare no competing financial interests.

This work was supported by National Eye Institute R01 EY-016407 and R01 EY-027925 to C.E.C., and New York University Global Seed Grants for Collaborative Research to K.K.S. and C.E.C. We thank Jeffrey Kravitz for processing and analyzing the eye tracking data; New York University's Center for Brain Imaging for technical support; and Martin Paré for helpful discussions about the research study.

Correspondence should be addressed to Kartik K. Sreenivasan at kartik.sreenivasan@nyu.edu.

https://doi.org/10.1523/JNEUROSCI.2016-20.2020

Copyright $\odot 2020$ Rahamati et al.

This is an open-access article distributed under the terms of the Creative Commons Attribution 4.0 International license, which permits unrestricted use, distribution and reproduction in any medium provided that the original work is properly attributed. feature space (Ma et al., 2014). Evidence from human studies using fMRI, which measures brain activity at scales that tile the space of neural population, supports the notion that macro-level distributed patterns of activity encode WM representations (Christophel et al., 2012; Riggall and Postle, 2012; Sreenivasan et al., 2014; Ester et al., 2015). Computational model-based methods designed to identify these information-containing patterns demonstrate that WM representations are present in a surprising number of cortical brain regions (Sreenivasan and D'Esposito, 2019). The success of these methods depends on both how encoding of features is distributed across a neural population (e.g., how visual space is distributed over V1) and how precisely aggregate voxelwise measures of neural activity are feature-tuned (Brouwer and Heeger, 2009; Naselaris et al., 2011). It follows that WM representations of visual space are robustly encoded in areas with systematic retinotopic organization, such as early visual cortex (Sprague et al., 2014; Rahmati et al., 2018), and to lesser extents in areas with coarse topography, such as frontoparietal cortex (Jerde et al., 2012; Mackey et al., 2017).

Here, we focus on a subcortical structure, the human superior colliculus (SC), and test its potential role in spatial WM. We are 
motivated by the growing appreciation that subcortical regions play key roles in cognition (Basso and May, 2017; Halassa and Kastner, 2017), specifically the role of SC in attention (Ignashchenkova et al., 2004; Katyal and Ress, 2014). Additionally, the well-established topography of the macaque SC (Cynader and Berman, 1972; Goldberg and Wurtz, 1972a) and advances in imaging the human SC (Krebs et al., 2010a,b; DeSimone et al., 2015; Furlan et al., 2015; Zhang et al., 2015; Savjani et al., 2018) suggest the recent developments in modeling fMRI measures of population activity can be a powerful tool to study the human SC.

Traditionally, the SC mediates orienting behaviors (e.g., gaze) by coordinating activity between two tightly registered eye-centered topographic maps: a map representing input from the visual system and a map representing motor output in the form of the angle and amplitude of saccades (Wurtz and Albano, 1980; Sparks, 2002; Gandhi and Katnani, 2011). However, two recent lines of evidence argue against a strictly visuomotor-centric model of SC function. First, pharmacological inactivation of the macaque SC motor map induces a form of visual neglect akin to extinction but does not cause anopsia (i.e., visual field defects) or paresis (i.e., deficits in voluntary oculomotion) (McPeek and Keller, 2004; Lovejoy and Krauzlis, 2010; Zénon and Krauzlis, 2012). Second, neural activity in the SC motor map encodes the spatiotopic locations of a behavioral goal, rather than the specific metrics of the saccade to the goal (Freedman and Sparks, 1997; Bergeron et al., 2003). Therefore, the functional role of the SC cannot be explained simply in terms of visual input or motor output. Instead, the SC may integrate signals computed throughout the brain into a common topographical organization, acting as a staging area for organizing goal-oriented behavior into a map of space weighted by bottom-up salience and top-down goal-relevance (Fecteau and Munoz, 2006). Indeed, studies that use memory-guided saccade (MGS) tasks to dissociate visual and saccade-related activity often observe delay period activity in macaque SC (Shen et al., 2011; Dash et al., 2015; Sadeh et al., 2018), consistent with a role in spatial WM.

The aims of our study were twofold. First, we used computational approaches to extend our understanding of the topography of human SC. Second, we leveraged information about SC's underlying architecture identified in our first aim to model how population activity in SC encodes WM representations disentangled from simple visual or motor components.

\section{Materials and Methods}

Participants. One female and five male subjects (ages 27-49 years; one left-handed) participated in the study. The subjects were in good health with no history of psychiatric or neurologic disorders, had normal or corrected-to-normal visual acuity, and gave informed written consent. The study was approved by the New York University Committee on Activities Involving Human Subjects and the New York University Abu Dhabi Institutional Review Board.

Stimulus display. We generated stimuli and interfaced with the MRI scanner, button-box, and eye-tracker using MATLAB software (The MathWorks) and Psychophysics Toolbox 3 (Brainard, 1997; Pelli, 1997). Stimuli were presented using a PROPixx DLP LED projector (VPixx Technologies) located outside the scanner room and projected through a waveguide and onto a translucent screen located at the end of the scanner bore. Subjects viewed the screen at a total viewing distance of $64 \mathrm{~cm}$ through a mirror attached to the head coil. The display subtended $\sim 32^{\circ}$ of visual angle horizontally and vertically. A trigger pulse from the scanner synchronized the onsets of stimulus presentation and image acquisition.

Eye tracking. To ensure fixation compliance and to record saccadic responses, we measured eye gaze constantly throughout the experiment using an MRI-compatible Eyelink 2K (SR Research). Using the freely available iEye toolbox (www.github.com/clayspacelab/iEye), we preprocessed and scored eye-tracking data automatically, quantified the accuracy (absolute Euclidian distance between the saccade landing point and the true target location), precision (average SD of tangential and radial components of the saccade landing points), and response times of visual and MGSs, and plotted example time-courses and trajectories of saccades shown in Figure $2 C, D$. Subjects were able to reliably fixate through the delay; fixation breaks (saccade amplitude $>2^{\circ}$ from fixation) during the delay only occurred in $0.5 \%-2.0 \%$ of the trials in 5 of 6 subjects. The remaining subject made quick saccades away from and then back to fixation on $7.0 \%$ of trials. Given the relative infrequency of unwanted saccades, we did not exclude any trials from the fMRI analyses.

MRI data acquisition and preprocessing. MRI data were acquired in the Center for Brain Imaging at New York University with a 3-Tesla Siemens Prisma MRI scanner using a 32-channel head coil. Twenty functional series of 120 volumes were collected for the retinotopic mapping task and 20 functional runs (except for 1 subject from whom we collected 10 runs) of 232 volumes were collected for the spatial WM task. Each functional run was acquired with 14 coronal slices and a gradient echo, echo planar sequence with a 128 square matrix, $192 \mathrm{~mm}$ field of view (FOV), and $2.0 \mathrm{~mm}$ slice thickness, leading to a voxel size of $1.5 \times 1.5 \times 2.0 \mathrm{~mm}\left(\mathrm{TR}=1.5 \mathrm{~s}, \mathrm{TE}=41 \mathrm{~ms}\right.$, flip angle $=66^{\circ}$, bandwidth $=$ $752 \mathrm{~Hz} /$ pixel). A partial Fourier factor of $7 / 8$ was used to acquire an asymmetric fraction of $k$ space and GRAPPA parallel imaging with a factor of 2 was used to reduce acquisition time. The posterior edge of the acquisition volume was aligned in the mid-sagittal plane with the posterior edge of inferior colliculus. We also collected a high-resolution $\mathrm{T}_{1}$-weighted MPRAGE $(0.8 \mathrm{~mm}$ isotropic voxels, $256 \times 240 \mathrm{~mm})$ to register functional scans to an anatomic image. In addition, for each scanning session, we collected a single whole-brain-coverage functional image ( $\mathrm{TR}=10.8 \mathrm{~s}$ ) with the same spatial resolution as the partial-brain coverage functional images to align the partial-coverage functional images to the whole-brain anatomic images. We motion-corrected the functional data through a rigid body ( 6 parameter) transform. After confirming that subject motion was limited $\left(\operatorname{mean}[\max ]\right.$ rotation $=0.13^{\circ}\left[0.39^{\circ}\right]$, mean $[\max ]$ translation $=$ $0.14[0.50] \mathrm{mm}$ ), we coregistered the functional data with the anatomic images according to the transformation calculated using the whole-braincoverage functional image. All functional data were kept in the original spatial and temporal resolution (no smoothing) for both WM and retinotopy analyses. Finally, we removed the linear trend and converted the time-series to $z$ units for each voxel.

Population receptive field ( $p R F)$ mapping. We used established procedures to model the pRF parameters in SC voxels (Dumoulin and Wandell, 2008; DeSimone et al., 2015). During scanning, subjects were presented with a black-and-white checkerboard-patterned bar whose elements reversed contrast with a full-cycle frequency of $8 \mathrm{~Hz}$ (Fig. 1A). The bar subtended $8^{\circ}$ of visual angle across its width and extended beyond the boundaries of the screen along its length. The bar was oriented either vertically or horizontally and swept across the screen perpendicular to the bar orientation, passing through central fixation. Each scanning run consisted of four $30 \mathrm{~s}$ sweeps (left to right, right to left, top to bottom, and bottom to top) in a random order, with $12 \mathrm{~s}$ mean-luminance blank periods at the start and end of the run. Subjects performed a demanding fixation task that required them to detect and map the color of the fixation cross (which could turn red, green, blue, or yellow every 1.5 s) to one of four button presses. For the mapping experiment, we modeled each voxel in terms of a Gaussian pRF (Fig. 1A) using methods and tools previously described (DeSimone et al., 2015, 2016). The pRF model provides a description of each voxel's BOLD response in terms of a retinotopic location and extent. We also modeled the delay of the hemodynamic response function (HRF) and the baseline of the BOLD signal (DeSimone et al., 2015; Thomas et al., 2015). The delay parameter estimates the time to peak and time to undershoot of the HRF. The baseline parameter ensures that the modeled and measured BOLD signals vary about a single global mean. In an initial phase of the parameter estimation, we used a sparse and coarse grid search with an effective stimulus downsampled by $2 \mathrm{D}$ bilinear interpolation to $5 \%$ of the original resolution. The best fit 
A

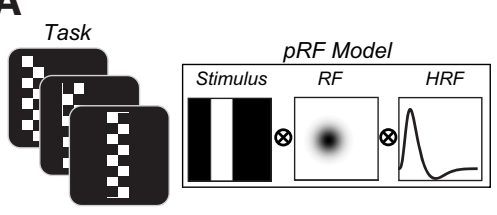

B

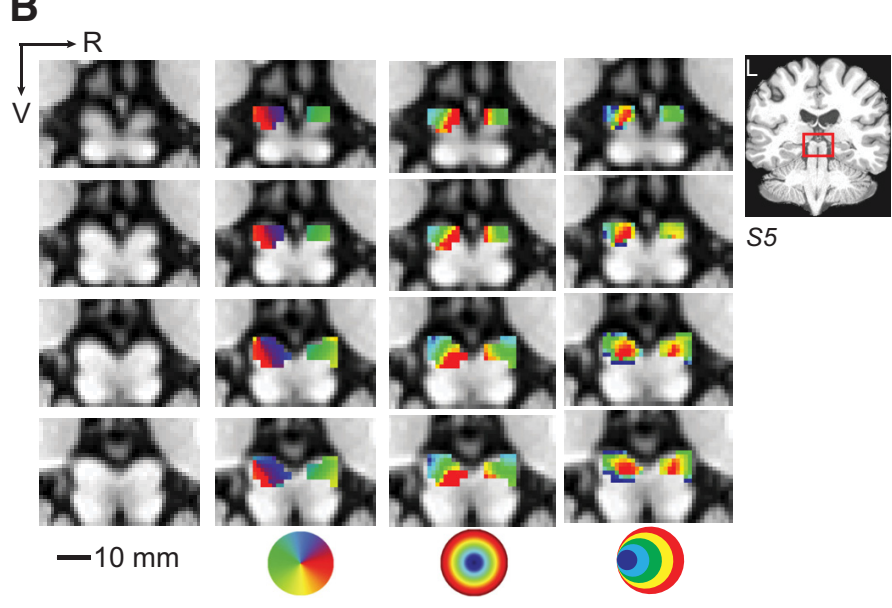

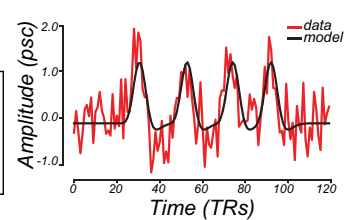

c $\Gamma^{\mathrm{R}}$
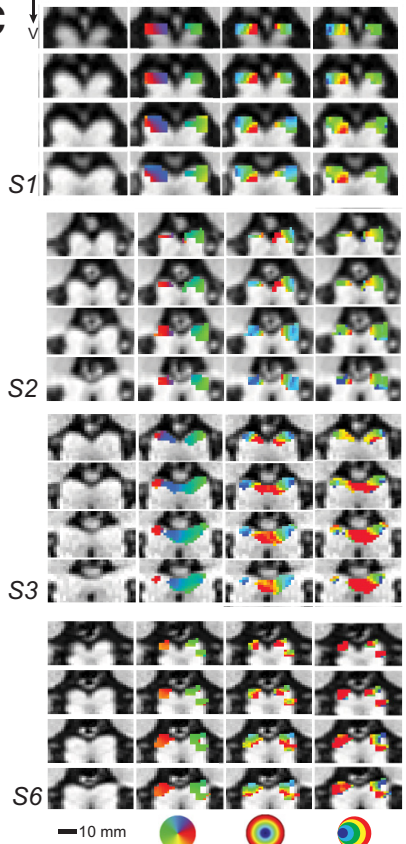

Figure 1. Topographic mapping of human SC. A, To model voxel pRFs, subjects viewed bars of contrast reversing checkerboards that swept across the visual field. Bar positions over time converted into binary apertures were projected onto a 2D Gaussian model of a receptive field (RF) and convolved with an HRF. Right, A single-sample voxel in SC is plotted for one run. $\boldsymbol{B}$, Enlarged coronal slices through the human SC in an example subject (red box inset). R, Right; V, ventral. Left to right, Columns represent the T1 anatomy, polar angle, eccentricity, and size parameter maps of an example subject (S5; thresholded at $\left.r^{2} \geq 0.1\right)$. Colored circles represent visual field keys. C, Topography of SC is consistent across other subjects.

from the sparse sampling of model parameter space was used to estimate the best fitting HRF parameters (delay to peak and undershoot), and then used as a seed in the final phase of a fine-tuned gradient-descent error minimization using the nonresampled stimulus.

For each subject, the SC ROI was drawn based on anatomy and a pRF model threshold of $r^{2} \geq 0.1$. The pRF model failed in 1 subject (S4), even when lowering the cutoff threshold, and we could not discern topography in SC. Thus, for this subject, we selected all voxels within the SC based on anatomic T1 images for further analysis. Importantly, our spatial WM results were not dependent on subject S4; indeed, the results were statistically more robust when excluding $\$ 4$, although we include $\mathrm{S} 4$ in the results presented below for completeness. Using procedures similar to Winawer et al. (2010), we estimated the FOV of the SC map from the full pRF model. To represent the FOV of the full SC map in visual space, we used 2D Gaussians whose positions within the visual field and widths were determined by each voxel's pRF center and size parameters, and whose maximum value equaled 1 . We did this on the pRF parameters aggregated across the left and right SC of all subjects. Since many points in the visual field were covered by several pRFs, when combining the pRFs, we mapped each visual field coordinate to the maximum $\mathrm{pRF}$ value.

Spatial WM experiment. In order to measure spatial WM representations in the SC, we imaged the brain while subjects maintained a location in WM during a long memory retention interval (see Fig. $3 A$ ). Trials began with a brief visual stimulus (full contrast circle with a radius of $0.25^{\circ}$ of visual angle) presented for $300 \mathrm{~ms}$ in the periphery at one of eight angular locations evenly spaced from $22.5^{\circ}$ to $337.5^{\circ}$ of polar angle in $45^{\circ}$ intervals and jittered by $\pm 10^{\circ}$, at an eccentricity of $9^{\circ}-11^{\circ}$ of visual angle (see Fig. $3 B$ ). The color of the visual stimulus indicated the transformation required to remap its location to the goal of a later MGS. A white stimulus indicated no transformation; green indicated an MGS to the location mirrored across the horizontal meridian; red indicated an MGS to the location mirrored across the vertical meridian; and blue indicated an MGS to the location mirrored across both the horizontal and vertical meridians. After a $10.5 \mathrm{~s}$ memory delay, a black dot appeared for $400 \mathrm{~ms}$ at a random uniformly sampled polar angle $\left(0^{\circ}-360^{\circ}\right)$ and radius $\left(9^{\circ}-11^{\circ}\right.$ of visual angle) from central fixation. Subjects first made a visually guided saccade (VGS) to this target, and then immediately made an MGS to the transformed location guided by memory (1.4 s allotted for both saccades). Finally, the stimulus was represented at the correct transformed location as a means of providing performance feedback $(500 \mathrm{~ms})$. After subjects made a corrective saccade to the feedback target, an intertrial interval followed of $9.8 \mathrm{~s}$ of central fixation that preceded the next trial. Each scanning run (10 per session) contained 16 trials (each $22.5 \mathrm{~s}$ ), sampling each of the 8 angular locations twice. The four MGS transformation conditions were counterbalanced across pairs of successive scanning runs, resulting in 80 trials per condition for 4 subjects ( 40 trials per condition for 2 subjects). Each scanning session lasted $58 \mathrm{~min}$. All subjects practiced one block of 16 trials outside of the scanner and one to two blocks in the scanner before the experiment began.

Two task manipulations were designed to help us understand the nature of the information maintained in the SC during the memory delay. First, the intermediate VGS prevented subjects from being able to plan, and potentially maintain, the metrics of the MGS during the delay. Second, the various transformations moved the task-relevant location, the goal of the MGS, to a position in visual space that was independent of the retinal position of the visual stimulus. Together, we aimed to eliminate simply visual and/or motor components while honing in on WM representations of visual space.

Inverted encoding model (IEM). To reconstruct a representation of spatial WM from the pattern of SC activity during the memory delay, we used a spatial IEM (Brouwer and Heeger, 2009). First, we modeled each voxel's response as a weighted sum of nine information channels, each in the form of a squared one-dimensional cosine function centered at one of the nine equally spaced polar angles around an invisible ring. We estimated voxel-channel weights by fitting a GLM to a subset of data used only for training. For this training, we only used trials in which the visual stimulus and MGS target were colocated ("Same location" condition, see Fig. 3A). We then inverted these regression weights to estimate the contribution of each channel to a representation of visual space in the held-out data from the other conditions that required a spatial transformation of the visual stimulus. Finally, we averaged all information channels, weighted by their estimated channel contribution, to reconstruct the population's representation. For visualization purposes, we 

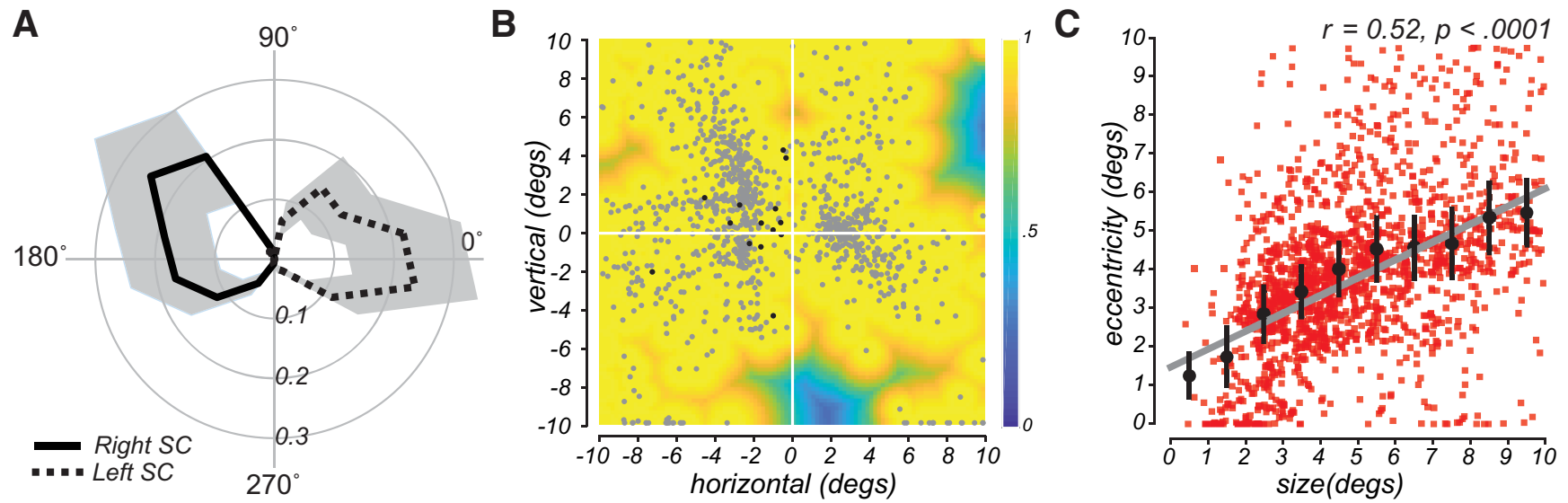

Figure 2. A, Radial histograms of pRF polar angle in SC demonstrate strong contralateral coverage of the visual field. Based on pRFs from all subjects, the lines are the mean fractional volume representing each polar angle ( \pm SEM). $\boldsymbol{B}$, Aggregate FOV when pRF location and size parameters are combined. Each gray dot represents the center of single-voxel pRFs. Color represents the maximum pRF value across the population of voxels in the $S C$ and reflects the relative effectiveness of visual stimulation in evoking a response in the $S C$. Black dots ( $n=14$ ) represent pRFs from the left SC with a center in the ipsilateral left visual field; no such ipsilateral centers were found in the right SC. C, Size of voxel pRFs in the SC increased linearly with eccentricity. Red squares represent SC voxels from all subjects. Black dots represent binned means ( $\pm S E M)$. Gray line indicates linear fit.

depict the information channels arranged around an invisible ring centered in the visual field. We estimated the population activity in each trial by averaging each voxel's BOLD activity during the last four TRs of the delay period. To increase the signal-to-noise ratio, we combined trials by computing a twofold mean trial time-series, reducing the total number of trials by half while maintaining the counterbalancing of the exemplars across the memory locations. We repeated the IEM training and reconstruction procedure using a 10,000 iteration bootstrap procedure with different arrangements of trials for computing the twofold mean time-series. This ensured that any effects were not simply because of bias in the sampling and recombination of trials.

Statistical analysis. To quantify the goodness of our reconstructions, we used a modified version of the representational fidelity metric (Sprague et al., 2016). Representational fidelity quantifies the similarity between a given reconstruction and a standard tuning function; however, this can be overly sensitive to the gain of the reconstruction peak at the cost of sensitivity to deviations from the reconstruction center. To adjust the sensitivity of the fidelity metric, we included a cost function in our modified fidelity calculation (Eq. 1), where $f_{\text {stndrd }}$ and $f_{\text {recon }}$ are the standard and reconstructed tunings (both circular), $l$ is the location $\left(0^{\circ}\right.$ $\left.360^{\circ}\right), g$ is the cost function, $\theta_{f_{s} s t n d r d}$ is the parameter set of the standard tuning function, and $\mathrm{err}_{\text {recon }}$ is the deviation (error) of the reconstruction peak from the true location.

$$
\text { Fidelity }=\left[f_{\text {stndrd }}(l) \cdot f_{\text {recon }}(l)\right] \times g\left(\theta_{f_{\text {stndrd }}}, \text { err recon }\right)
$$

To validate the significance of our reconstructions, we built 10,000 IEMs, each trained after shuffling the training data, and compared the fidelity distributions corresponding to the real and permuted data through a nonparametric Kolmogorov-Smirnov test at the individual subject level and a paired $t$ test, after a 1000 iteration bootstrap across subjects, at the group level.

In order to link the pRF model of retinotopy and the spatial IEM, we compared each voxel's polar angle preference derived from the two models. For the pRF model, we simply used the polar angle of the pRF center. For the IEM, we summed all information channels weighted by their estimated regression coefficients yielding a polar angle tuning curve for each voxel. Since the IEM estimates were derived from the task in which all stimuli were $9^{\circ}-11^{\circ}$ in the periphery, we restricted our analysis to SC voxels whose pRF centers were at least $5^{\circ}$ in eccentricity. We then calculated the circular correlation coefficient between the pRF polar angle and the peak of the IEM tuning curve.

Data availability. Experimental data and analysis code are available at https://osf.io/mkgd2/.

\section{Results}

\section{Retinotopic mapping}

Following our pRF mapping procedures (Fig. $1 \mathrm{~A}$ ), we examined the modeled RF properties of voxels in the SC by overlaying the pRF model parameters on the T1 anatomic image (shown for a representative subject in Fig. $1 B$ and individual subjects in Fig. $1 C)$. We found orthogonal polar angle and eccentricity representations of the visual field along the SC. The topography revealed a graded upper-to-lower visual field representation along the medial-to-lateral axis of the SC, and a graded foveal-to-peripheral visual field representation along the anterior-to-posterior axis. The SC pRFs largely tiled the contralateral visual field (Fig. $2 A)$. The "bow-tie" shape of the distribution of polar angles, as found in previous fMRI studies of the retinotopy of the SC (Schneider and Kastner, 2005; DeSimone et al., 2015), seems to imply a relative underrepresentation of angles near the vertical meridian. However, when we consider the full RF model and combine the pRF centers and sizes to estimate the FOV of the $\mathrm{SC}$, it is clear that visual stimulation at most retinal locations effectively drives the SC (Fig. $2 B$ ). Moreover, as in the macaque (Marino et al., 2008), we found a positive correlation between the size and eccentricity of pRF parameters in the SC (Pearson's $r=0.52, p<0.0001$; Fig. $2 C$ ). Overall, our model of the topographic structure of the human SC closely resembles that of the cat and macaque (Cynader and Berman, 1972; Goldberg and Wurtz, 1972a) and a previous report in humans (DeSimone et al., 2015).

\section{Spatial WM}

The accuracy, precision, and latency of visual and MGSs were similar to previous studies that used delayed saccade or antisaccade tasks (Curtis and Connolly, 2008; Saber et al., 2015; Montez et al., 2017), indicating that subjects could perform the task well despite the transformations and double saccades (Fig. 3C,D). Performance did not differ across task conditions (one-way repeated-measures ANOVAs, all $p$ values $>0.4$ ). The VGSs were slightly longer in latency than previous reports (compare Curtis and Connolly, 2008), perhaps owing to the long delay, complexity of the task, or the simultaneous task of remembering the future MGS target.

The average BOLD signal in the retinotopically defined human SC persisted above pretrial baseline during the memory 
A

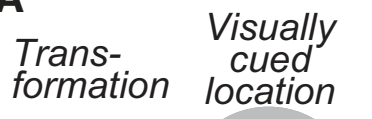

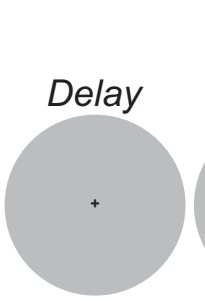
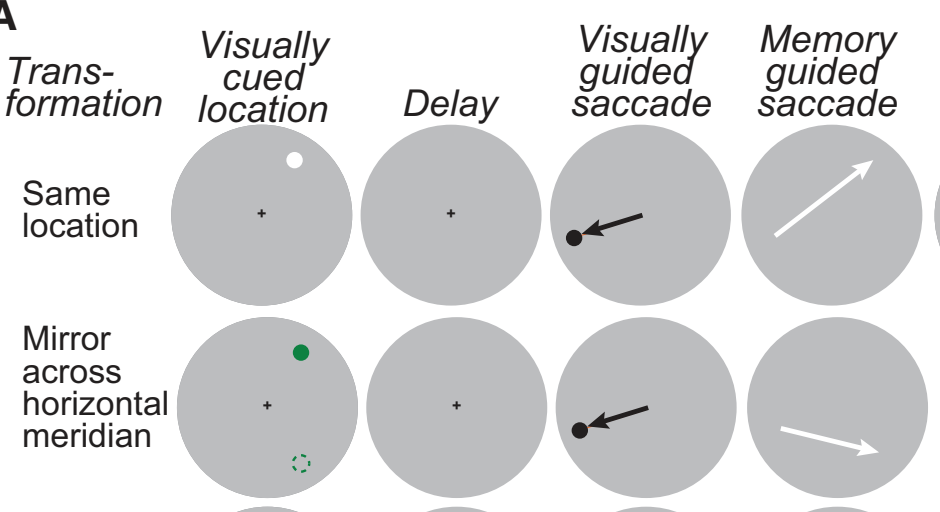

Mirror

across

meridian
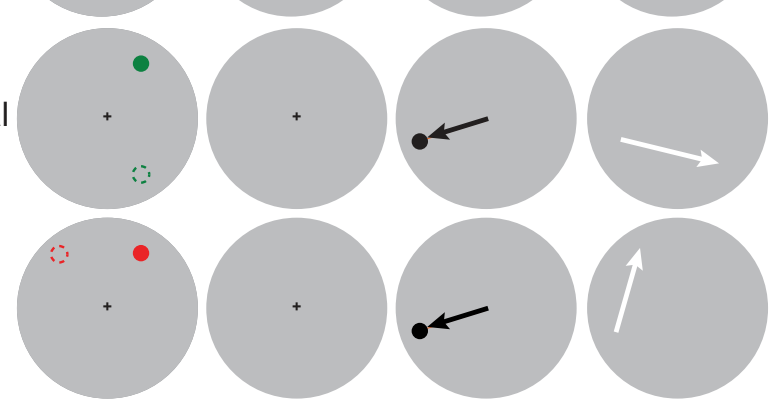

Mirror

across

both

meridians
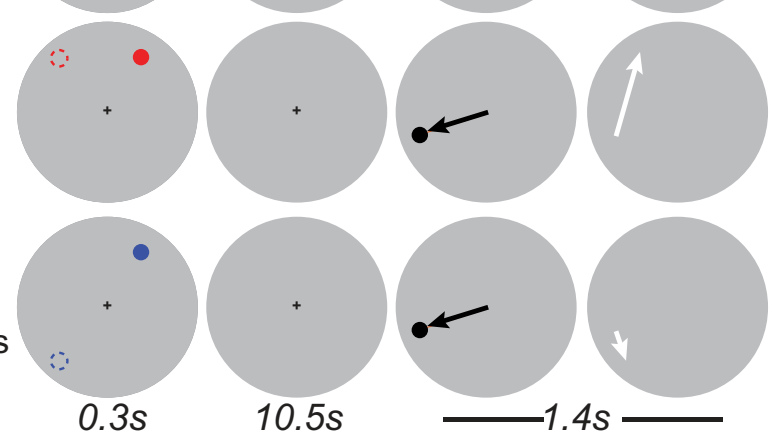

D

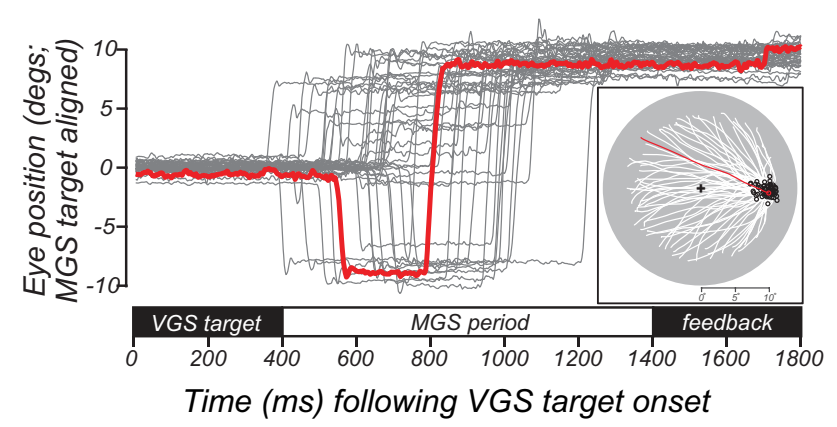

B
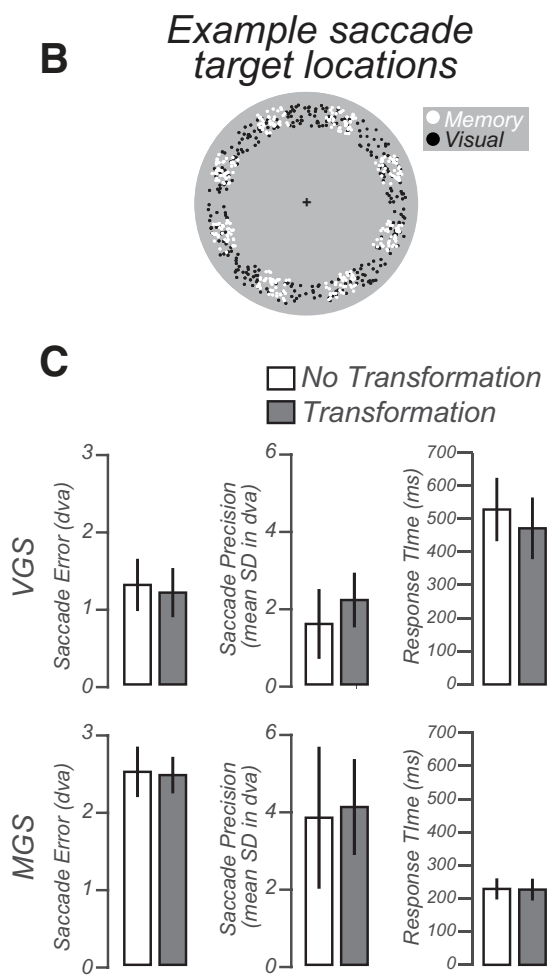
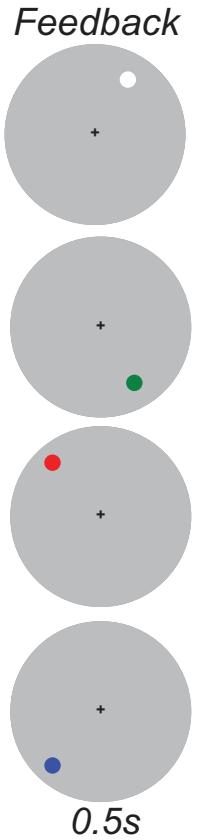

E

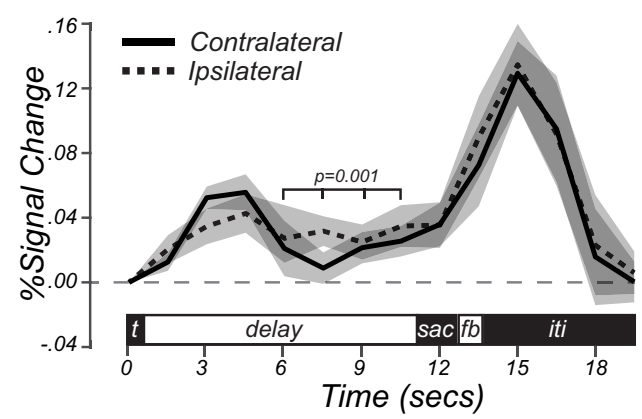

Figure 3. Task schematic, behavioral data, and delay-period activity in human SC. A, Schematic of four types of MGS trials. In each condition, trials began with a brief visual target located in the periphery (colored dots; left column). Following a delay, subjects made a VGS to a target whose location was unpredictable. Then, subjects immediately made an MGS to a location based on the initial visual target. In one condition, the MGS was directed to the visual target. In the other conditions, the MGS was made to simple geometric transformations of the visual target (dashed circles, left column; for reference here but not displayed). These included mirror transformations across each meridian and both meridians. The color of the visually cued target represented the type of transformation. Feedback was provided after the MGS with a visual stimulus at the correct location. Because of the VGS, the metrics of the MGS could not be predicted. The transformations dissociated the goal of the MGS from the visually stimulated retinal position. $\boldsymbol{B}$, Locations of VGS and MGS targets were distributed $9^{\circ}-11^{\circ}$ in the periphery. $\boldsymbol{C}$, Median saccade accuracy, precision, and response time for VGS (top) and MGS (bottom) plotted separately for the trials in which there was no transformation of the visual target (same; white) and the trials in which the visual target required a mirrored transformation (i.e., trials with transformations across horizontal, vertical, and both meridians are collapsed). There were no statistically significant differences between the transformed and untransformed trials for any of the saccade metrics. Error bars indicate SEM across subjects. D, Example eye-tracking traces (gray lines) from a subject during one scanner session. All trials are rotated such that, despite the various transformations of the visual target location, all MGS targets are rotated to a location $10^{\circ}$ to the right. Red trace represents an example trial. Inset, We replot only the MGS trajectories (white lines), which start from a wide variety of peripheral locations following the VGS but converge and end (black circles) near the aligned MGS target location. Red trace represents an example trial. $\boldsymbol{E}$, Group-averaged ( \pm SEM) BOLD signal in SC voxels persisted significantly above pretrial baseline (gray dashed line) during the delay period for trials when the MGS target was in the contralateral and ipsilateral hemifield. The delay was defined as the average of the last four TRs in the delay period, identified by the bracket above the time courses.

period, suggesting that it may play an important role in WM (percent signal change; mean $=0.04,95 \% \mathrm{CI}=[0.009,0.08]$, onetailed Student's $t$ test, $p<0.01$; Fig. $3 E$ ). However, it was not lateralized with respect to the MGS target, a pattern incongruous with the clear lateralization of the SC pRFs (Figs. 1, 2). There are several possible explanations for the lack of lateralization: delay activity may be contaminated by the earlier visual stimulation, it may reflect subjects' covert attention to the entire visual field in anticipation of the intervening VGS target, or it may result from the complex transformations required by the task. Alternatively, averaging over many voxels may result in a measure that is too coarse to capture the population dynamics by which the SC encodes WM (Furlan et al., 2015).

Because of these uncertainties and motivated by our pRF findings, we used a multivoxel model of visual space (Fig. 4) to test whether topographic patterns of activity in human SC encode WM locations. Conceptually, the model provides a means to map a multivoxel population response into the coordinates of visual space. We assumed an underlying neural architecture based on the retinotopic organization of the voxels within 

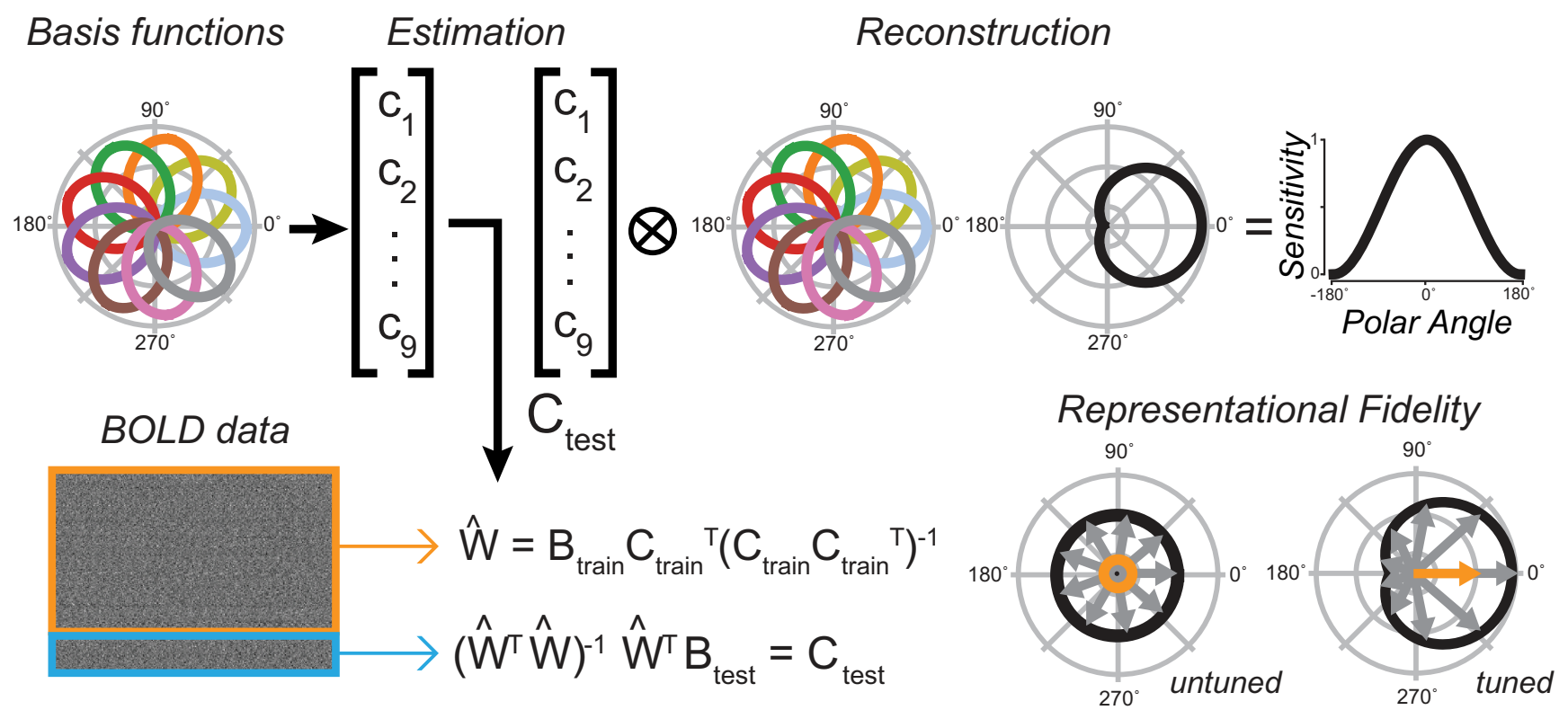

Figure 4. IEM. Using a standard IEM, we calculated regression weights (W) from a training set of BOLD data ( $B_{\text {train; }}$ orange box) and corresponding hypothetical channel coefficients ( $\left.C_{\text {train }}\right)$ represented by nine evenly spaced radial basis functions, each tuned to a specific angle. We calculated the contribution of each basis function in the final reconstruction $\left(C_{\text {test }}\right)$ by linearly combining a new set of BOLD data ( $B_{\text {testi }}$ blue box) and the inverse of the regression weights. To reconstruct a representation of visual space, we used a linear combination of all basis functions, each weighted by its corresponding contribution in $C_{\text {test. }}$. Right, We unwrap the curve to show a sample sensitivity profile across angles in visual space. We calculated representational fidelity, a metric for the goodness of reconstructions, as the vector mean of a set of unit vectors around different angles, each weighted by the reconstructed sensitivity at that position. Displayed are examples of poor/untuned and good/tuned representations. Conceptually, our model provides a means to map a multivoxel population response into the coordinates of visual space.

SC, and modeled each voxel's response with a set of basis functions that tiled polar angle space. We tested this assumption by comparing the angles derived from the pRF model with the angles derived from the IEM for each voxel in the SC. First, we trained the model using trials in which no transformation of the visually cued target was required, deriving modeled basis functions from which we estimated each voxel's preferred polar angle. Second, using circular correlation, we confirmed that the pRF and IEM polar angle parameters were similar $(r=0.26, p<0.002)$. This suggests that the two forward-modeling approaches converged on very similar polar angle parameters despite the differences in modeling (pRF and IEM) and differences in cognitive demands (visuospatial attention and WM). Next, using the IEM-based model trained on "Same location" trials, we tested it on trials requiring transformations (see Materials and Methods). Consistent with the notion that SC population delay activity encodes spatial information in WM, our model could accurately reconstruct the transformed location of the MGS (Fig. $5 A$, right). Importantly, these locations stored in WM were computed from spatial transformations of the visual targets and thus were not locations that were retinally stimulated earlier in the trial. Models trained on the location of the visual target or the VGS location were unable to reconstruct these locations (Fig. 5A, left, middle), indicating that SC delay activity encoded the abstract representation of the memory location rather than the visually presented targets. Quantification of these results using our modified representational fidelity metric confirmed that SC population activity during the delay was spatially tuned only for the location of the MGS target (subject mean fidelity $=0.0013$, $95 \% \mathrm{CI}=[0.0010,0.0023]$, one-tailed Student's $t$ test between real and permuted reconstructions, $p<0.001$; Fig. $5 B$ ). Remarkably, we found these effects at the individual level in every subject except S4, the subject in which we could not discern topography in the SC based on our pRF model (Fig. 5C). Overall, the results were consistent and provide robust evidence for spatial WM encoding in topographically organized human SC.

\section{Discussion}

Motivated by the increasing role that the subcortex (Halassa and Kastner, 2017) and specifically the SC (Basso and May, 2017) is thought to play in cognition, we used recent developments in fMRI modeling (Serences and Saproo, 2012) to test how WM representations are encoded in the population activity of the human SC. We found that the topography of human SC resembled that of macaque SC, the activity in retinotopic SC persisted during WM retention intervals, and, at the population level, it encoded the spatial location of WM representations.

\section{Retinotopy of human SC}

Using pRF mapping, we identified a visual field map in human SC that systematically represented the contralateral visual field. Representations of upper and lower portions of visual fields were found in the medial and lateral, respectively, parts of the SC map. The representations of foveal and peripheral portions of the visual field were found in the anterior and posterior, respectively, parts of the SC map. Similar to reports in human visual cortex (Dumoulin and Wandell, 2008), we found that the size of the estimated RF of voxels correlated with its eccentricity, where smaller RFs were nearer the fovea. Both of these observations matched those reported using electrophysiology in macaques (Cynader and Berman, 1972; Goldberg and Wurtz, 1972a; Marino et al., 2008). Previous fMRI studies using phase-encoding methods for retinotopic mapping (Schneider and Kastner, 2005, 2009; Katyal et al., 2010) or derived during the generation of saccades of different angles (Savjani et al., 2018) have found similar results with respect to the orderly contralateral maps of polar angle, suggesting that mapping evoked by saccades and visual stimulation is in registration or perhaps originates from the 

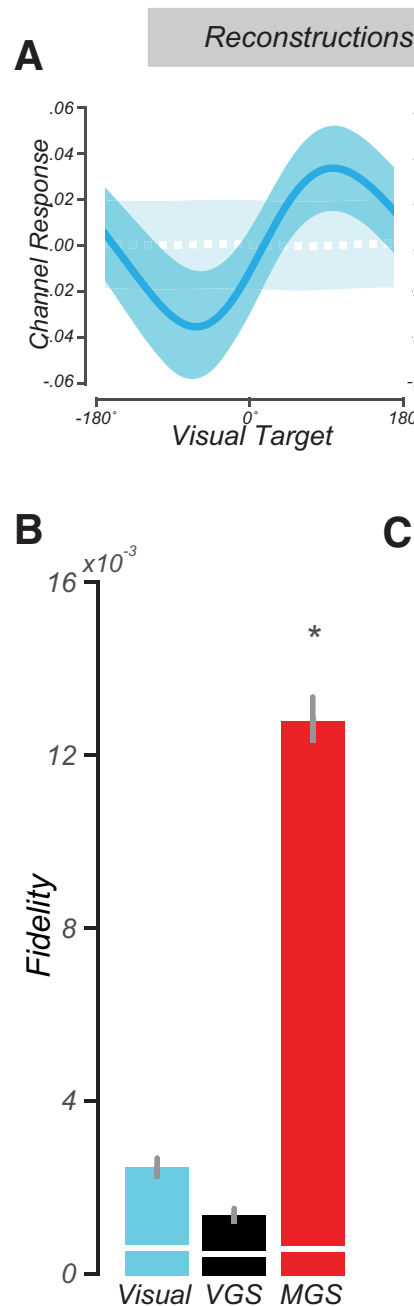

Reference Location

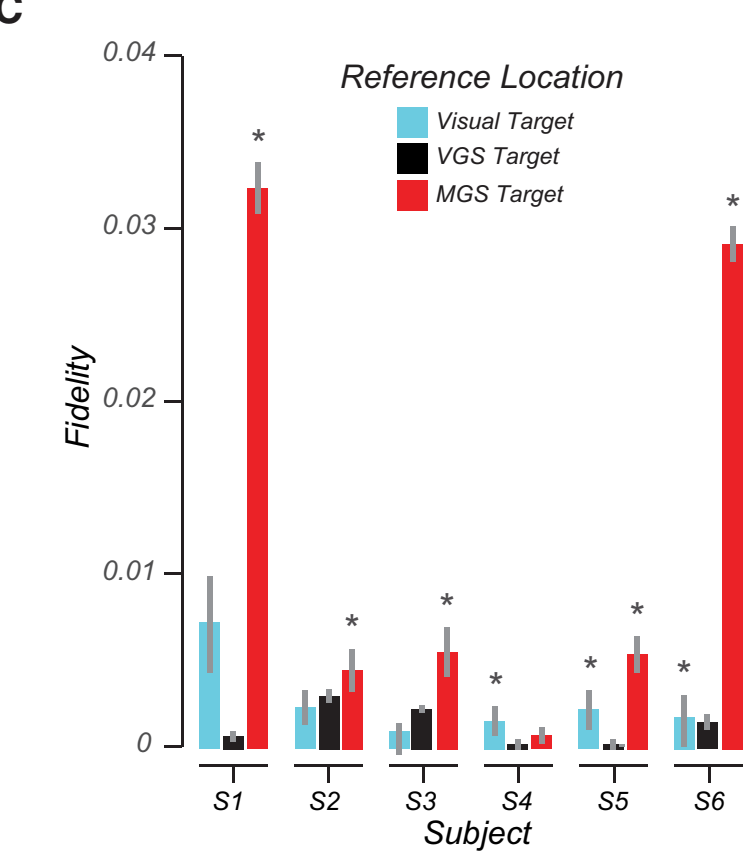

Figure 5. Modeling WM representations in human SC. A, We used delay period activity in human SC to reconstruct visua space. From left to right: the average reconstructed sensitivity ( \pm SEM) in visual space aligned to the visually cued target, VGS target, and MGS target locations, respectively. In each panel, all trials are aligned to the corresponding reference location centered at $0^{\circ}$. Dashed white lines indicate reconstructions from BOLD data with the trial labels permuted. $\boldsymbol{B}$, Representational fidelity ( \pm SEM) corresponding to three reference locations, compared with shuffled data (white lines) computed at the group level. The SC population activity during the delay is largely tuned for the visual-spatial location of the MGS $\left(p<10^{-18}\right)$, not the visual target or VGS. C, Even at the individual subject level, we find greater fidelity for the MGS location for all subjects, except the 1 subject whose pRF model failed (S4). In 3 subjects, there was smaller tuning for the visually cued target, but this small effect was not significant at the group level. Asterisk indicate $p<0.01$.

same map. These studies also reported the same anisotropic distribution of angles we observed, with a lesser representation along the upper and lower vertical meridians compared with the horizontal meridian. The cause and significance of the anisotropy, which are also ubiquitous in cortical retinotopic areas, remain a point of debate (Larsson and Heeger, 2006; Winawer et al., 2010; DeSimone et al., 2015). Importantly, we add to previous characterizations of the human SC by mapping eccentricity along its rostral-caudal axis, mapping the RF size of its voxels, and using the full pRF model to estimate the FOV of the SC, which clearly covers the whole visual field.

\section{Spatial WM}

During the WM delay period, BOLD activity in SC was low but clearly persisted above pretrial baseline throughout the delay. On the one hand, this appears to align well with electrophysiological recordings from macaque SC neurons that typically show a slow but increased rate of discharge before saccades, including MGSs (Paré and Wurtz, 2001; Shen et al., 2011). On the other hand, the delay activity was not contralateralized with respect to the location of WM targets as would be expected based on the discharge properties of macaque SC neurons (Robinson, 1972) and the contralateral organization of the SC map in our subjects. This may be because of the complex visuomotor transformations required by our task. Alternatively, our coarse averaging of BOLD signals across all voxels from each side of the SC may comprise distributed neural activity related to foveating the central fixation stimulus (Krauzlis et al., 2017), general preparatory processes (Furlan et al., 2015), and/or inhibitory neural activity related to suppressing unwanted saccades to the remembered location during the delay (Ikeda et al., 2015). Although the delay activity suggests that human SC plays some role in spatial WM, an important finding in itself, it does not tell us precisely how the SC might contribute to WM.

Therefore, motivated by the spatiotopic organization of the SC, we constructed a multivoxel model of how population activity in SC encodes spatial WM representations. Similar encoding models of fMRI data have been useful in testing hypotheses about how cortical areas store relevant features of WM representations (Ester et al., 2015; Rahmati et al., 2018; Cai et al., 2019). Our results demonstrate that these models also work well in the subcortex, as we were able to model the population response in the SC that encoded spatial WM representations. Critically, the patterns of delay period activity we modeled did not encode retinal positions of past visual stimuli or future planned saccades. The locations held in WM were abstract transformations of visually stimulated locations, and the VGSs negated strategies involving the maintenance of saccade motor metrics. Therefore, the pattern of activity across the human SC neural population encodes abstract, cognitively defined locations in the absence of visual stimulation or motor commands. Thus, it is tempting to conclude that the SC signals driving our results originate from the intermediate and/or deep layers of the SC, where more cognitive processing is thought to occur (Ignashchenkova et al., 2004; White et al., 2017a). Moreover, neurons in the intermediate/deep, but not superficial, SC show persistent activity during MGS delays (Paré and Wurtz, 2001). However, the spatial resolution of our measurements precludes us from drawing firm conclusions about the layer specificity of our results.

The SC encoding of abstract locations in WM may be initiated by feedback signals from the cortex. If so, we might ask: what is the nature of these signals and from where do they originate? With respect to their nature, they resemble the spatial 
attention effects that have been described in the macaque SC (Krauzlis et al., 2013). Visually evoked SC responses are larger when the stimulus is behaviorally relevant and the goal of a saccade (Goldberg and Wurtz, 1972b). When task-related saccades are dissociated from the locus of attention, SC neurons with RF matching an attended target also show enhanced discharge rates (Ignashchenkova et al., 2004), and manipulations using microstimulation and chemical inactivation provide causal support for the role of the macaque SC in covert attention (Cavanaugh et al., 2006; Lovejoy and Krauzlis, 2010). Attention also causes enhanced neural responses measured with fMRI in the human SC (Schneider and Kastner, 2009; Katyal et al., 2010; Katyal and Ress, 2013). Indeed, one of the key mechanisms supporting spatial WM may involve sustained covert attention (Awh and Jonides, 2001; Jerde et al., 2012). In the context of our results, therefore, attention-related signals targeting neurons with RFs matching the transformed locations may sculpt the population encoded responses in the SC we observed. Likely sources of these top-down influences include brain areas with direct connections to the macaque SC, namely, lateral PFC, frontal eye field, lateral intraparietal area, and V1, each of which is known to support spatial WM functions (Sommer and Wurtz, 2001; Armstrong et al., 2009; Koval et al., 2011; Everling and Johnston, 2013; van Kerkoerle et al., 2017). Moreover, human fMRI studies using encoding models like the one used here consistently report that spatial WM representations are encoded in the patterns of population activity in these same cortical regions (Jerde et al., 2012; Riggall and Postle, 2012; Ester et al., 2015; Rahmati et al., 2018).

However, for a number of reasons, it is unlikely that SC simply integrates cortical commands and relays them to brainstem oculomotor plants. In the macaque, representations of visual priority emerge more rapidly in SC than in V1, indicating that feedback signals from SC may sculpt the gain of responses in cortex (White et al., 2017b). The SC has more ascending projections through the pulvinar and mediodorsal thalamus that could influence cortex, for example, than descending projections arriving into the SC (May, 2006). Moreover, lesions to the SC impair behaviors that depend on covert attention, but surprisingly do not affect the typical attentional enhancement of neuronal activity in extrastriate cortex (Zénon and Krauzlis, 2012). Therefore, the SC may play critical roles in spatial cognition, such as attention and WM, through circuits that both interact with but at the same time are independent of the cortex.

Our results depended on a model of the well-defined spatial topography of the SC, where space is systematically distributed over hundreds of voxels, and an assumption that WM was encoded in the SC population response. Indeed, we have long appreciated that the population of SC neurons collectively codes for visual and motor behavior (Lee et al., 1988; McIlwain, 1991), but the actual mechanism by which the population activity is combined remains controversial. As suggested by computational models of perception that posit neural populations encode a probability distribution over sensory features (Ma et al., 2006), the population response in SC may encode the probability of a prioritized, including a remembered, location (Fecteau and Munoz, 2006; Kim and Basso, 2010). Bayesian decoding models of fMRI population responses have provided compelling evidence for probabilistic encoding in visual cortex (van Bergen et al., 2015), which could be extended to the SC. Overall, future research should address how cortical and subcortical brain areas differ in their support of WM. Perhaps, they use similar mechanisms, but differences may emerge because of their areal inputoutput connections, parameters that govern local circuit-level dynamics, or broader factors related to behavioral goals and task contexts (Sreenivasan and D'Esposito, 2019).

\section{References}

Armstrong KM, Chang MH, Moore T (2009) Selection and maintenance of spatial information by frontal eye field neurons. J Neurosci 29:1562115629 .

Awh E, Jonides J (2001) Overlapping mechanisms of attention and spatial working memory. Trends Cogn Sci 5:119-126.

Basso MA, May PJ (2017) Circuits for action and cognition: a view from the superior colliculus. Annu Rev Vis Sci 3:197-226.

Bergeron A, Matsuo S, Guitton D (2003) Superior colliculus encodes distance to target, not saccade amplitude, in multi-step gaze shifts. Nat Neurosci 6:404-413.

Brainard DH (1997) The Psychophysics Toolbox. Spat Vis 10:433-436.

Brouwer GJ, Heeger DJ (2009) Decoding and reconstructing color from responses in human visual cortex. J Neurosci 29:13992-14003.

Cai Y, Sheldon AD, Yu Q, Postle BR (2019) Overlapping and distinct contributions of stimulus location and of spatial context to nonspatial visual short-term memory. J Neurophysiol 121:1222-1231.

Cavanaugh J, Alvarez BD, Wurtz RH (2006) Enhanced performance with brain stimulation: attentional shift or visual cue? J Neurosci 26:1134711358.

Christophel TB, Hebart MN, Haynes JD (2012) Decoding the contents of visual short-term memory from human visual and parietal cortex. J Neurosci 32:12983-12989.

Curtis CE, Connolly JD (2008) Saccade preparation signals in the human frontal and parietal cortices. J Neurophysiol 99:133-145.

Cynader M, Berman N (1972) Receptive-field organization of monkey superior colliculus. J Neurophysiol 35:187-201.

Dash S, Yan X, Wang H, Crawford JD (2015) Continuous updating of visuospatial memory in superior colliculus during slow eye movements. Curr Biol 25:267-274.

DeSimone K, Viviano JD, Schneider KA (2015) Population receptive field estimation reveals new retinotopic maps in human subcortex. J Neurosci 35:9836-9847.

DeSimone K, Rokem A, Schneider K (2016) popeye: a population receptive field estimation tool. J Open Source Softw 1:103.

Dumoulin SO, Wandell BA (2008) Population receptive field estimates in human visual cortex. Neuroimage 39:647-660.

Ester EF, Sprague TC, Serences JT (2015) Parietal and frontal cortex encode stimulus-specific mnemonic representations during visual working memory. Neuron 87:893-905.

Everling S, Johnston K (2013) Control of the superior colliculus by the lateral prefrontal cortex. Philos Trans R Soc Lond B Biol Sci 368:20130068.

Fecteau JH, Munoz DP (2006) Salience, relevance, and firing: a priority map for target selection. Trends Cogn Sci 10:382-390.

Freedman EG, Sparks DL (1997) Eye-head coordination during head-unrestrained gaze shifts in rhesus monkeys. J Neurophysiol 77:2328-2348.

Funahashi S, Bruce CJ, Goldman-Rakic PS (1989) Mnemonic coding of visual space in the monkey's dorsolateral prefrontal cortex. J Neurophysiol 61:331-349.

Furlan M, Smith AT, Walker R (2015) Activity in the human superior colliculus relating to endogenous saccade preparation and execution. J Neurophysiol 114:1048-1058.

Gandhi NJ, Katnani HA (2011) Motor functions of the superior colliculus. Annu Rev Neurosci 34:205-231.

Goldberg ME, Wurtz RH (1972a) Activity of superior colliculus in behaving monkey: I. Visual receptive fields of single neurons. J Neurophysiol 35:542-559.

Goldberg ME, Wurtz RH (1972b) Activity of superior colliculus in behaving monkey: II. Effect of attention on neuronal responses. J Neurophysiol 35:560-574.

Halassa MM, Kastner S (2017) Thalamic functions in distributed cognitive control. Nat Neurosci 20:1669-1679.

Ignashchenkova A, Dicke PW, Haarmeier T, Thier P (2004) Neuron-specific contribution of the superior colliculus to overt and covert shifts of attention. Nat Neurosci 7:56-64.

Ikeda T, Boehnke SE, Marino RA, White BJ, Wang CA, Levy R, Munoz DP (2015) Spatio-temporal response properties of local field potentials in the primate superior colliculus. Eur J Neurosci 41:856-865. 
Jerde TA, Merriam EP, Riggall AC, Hedges JH, Curtis CE (2012) Prioritized maps of space in human frontoparietal cortex. J Neurosci 32:1738217390.

Katyal S, Ress D (2013) Attentional base response in intermediate layers of human superior colliculus measured using high-resolution fMRI. J Vis 13:224.

Katyal S, Ress D (2014) Endogenous attention signals evoked by threshold contrast detection in human superior colliculus. J Neurosci 34:892-900.

Katyal S, Zughni S, Greene C, Ress D (2010) Topography of covert visual attention in human superior colliculus. J Neurophysiol 104:3074-3083.

Kim B, Basso MA (2010) A probabilistic strategy for understanding action selection. J Neurosci 30:2340-2355.

Koval MJ, Lomber SG, Everling S (2011) Prefrontal cortex deactivation in macaques alters activity in the superior colliculus and impairs voluntary control of saccades. J Neurosci 31:8659-8668.

Krauzlis RJ, Lovejoy LP, Zénon A (2013) Superior colliculus and visual spatial attention. Annu Rev Neurosci 36:165-182.

Krauzlis RJ, Goffart L Hafed ZM (2017) Neuronal control of fixation and fixational eye movements. Philos Trans R Soc Lond B Biol Sci 372:20160205.

Krebs RM, Schoenfeld MA, Boehler CN, Song AW, Woldorff MG (2010a) The saccadic re-centering bias is associated with activity changes in the human superior colliculus. Front Hum Neurosci 4:193.

Krebs RM, Woldorff MG, Tempelmann C, Bodammer N, Noesselt T, Boehler CN, Scheich H, Hopf JM, Duzel E, Heinze HJ, Schoenfeld MA (2010b) High-field FMRI reveals brain activation patterns underlying saccade execution in the human superior colliculus. PLoS One 5:e8691.

Larsson J, Heeger DJ (2006) Two retinotopic visual areas in human lateral occipital cortex. J Neurosci 26:13128-13142.

Lee C, Rohrer WH, Sparks DL (1988) Population coding of saccadic eye movements by neurons in the superior colliculus. Nature 332:357-360.

Lovejoy LP, Krauzlis RJ (2010) Inactivation of primate superior colliculus impairs covert selection of signals for perceptual judgments. Nat Neurosci 13:261-266

Ma WJ, Beck JM, Latham PE, Pouget A (2006) Bayesian inference with probabilistic population codes. Nat Neurosci 9:1432-1438.

Ma WJ, Husain M, Bays PM (2014) Changing concepts of working memory. Nat Neurosci 17:347-356.

Mackey WE, Winawer J, Curtis CE (2017) Visual field map clusters in human frontoparietal cortex. Elife 6:e22974

Marino RA, Rodgers CK, Levy R, Munoz DP (2008) Spatial relationships of visuomotor transformations in the superior colliculus map. J Neurophysiol 100:2564-2576.

May PJ (2006) The mammalian superior colliculus: laminar structure and connections. Prog Brain Res 151:321-378.

Mcllwain JT (1991) Distributed spatial coding in the superior colliculus: a review. Vis Neurosci 6:3-13.

McPeek RM, Keller EL (2004) Deficits in saccade target selection after inactivation of superior colliculus. Nat Neurosci 7:757-763.

Miller EK, Erickson CA, Desimone R (1996) Neural mechanisms of visual working memory in prefrontal cortex of the macaque. J Neurosci 16:5154-5167.

Montez DF, Calabro FJ, Luna B (2017) The expression of established cognitive brain states stabilizes with working memory development. Elife 6: e25606.

Naselaris T, Kay KN, Nishimoto S, Gallant JL (2011) Encoding and decoding in fMRI. Neuroimage 56:400-410.

Paré M, Wurtz RH (2001) Progression in neuronal processing for saccadic eye movements from parietal cortex area lip to superior colliculus. J Neurophysiol 85:2545-2562.

Pelli DG (1997) The VideoToolbox software for visual psychophysics: transforming numbers into movies. Spat Vis 10:437-442.

Rahmati M, Saber GT, Curtis CE (2018) Population dynamics of early visual cortex during working memory. J Cogn Neurosci 30:219-233.
Riggall AC, Postle BR (2012) The relationship between working memory storage and elevated activity as measured with functional magnetic resonance imaging. J Neurosci 32:12990-12998.

Robinson DA (1972) Eye movements evoked by collicular stimulation in the alert monkey. Vision Res 12:1795-1808.

Saber GT, Pestilli F, Curtis CE (2015) Saccade planning evokes topographically specific activity in the dorsal and ventral streams. J Neurosci 35:245-252.

Sadeh M, Sajad A, Wang H, Yan X, Crawford JD (2018) The influence of a memory delay on spatial coding in the superior colliculus: is visual always visual and motor always motor? Front Neural Circuits 12:74.

Savjani RR, Katyal S, Halfen E, Kim JH, Ress D (2018) Polar-angle representation of saccadic eye movements in human superior colliculus. Neuroimage 171:199-208.

Schneider KA, Kastner S (2005) Visual responses of the human superior colliculus: a high-resolution functional magnetic resonance imaging study. J Neurophysiol 94:2491-2503.

Schneider KA, Kastner S (2009) Effects of sustained spatial attention in the human lateral geniculate nucleus and superior colliculus. J Neurosci 29:1784-1795.

Serences JT, Saproo S (2012) Computational advances towards linking BOLD and behavior. Neuropsychologia 50:435-446.

Shen K, Valero J, Day GS, Paré M (2011) Investigating the role of the superior colliculus in active vision with the visual search paradigm. Eur J Neurosci 33:2003-2016.

Sommer MA, Wurtz RH (2001) Frontal eye field sends delay activity related to movement, memory, and vision to the superior colliculus. J Neurophysiol 85:1673-1685.

Sparks DL (2002) The brainstem control of saccadic eye movements. Nat Rev Neurosci 3:952-964.

Sprague TC, Ester EF, Serences JT (2014) Reconstructions of information in visual spatial working memory degrade with memory load. Curr Biol 24:2174-2180.

Sprague TC, Ester EF, Serences JT (2016) Restoring latent visual working memory representations in human cortex. Neuron 91:694-707.

Sreenivasan KK, D'Esposito M (2019) The what, where and how of delay activity. Nat Rev Neurosci 20:466-481.

Sreenivasan KK, Vytlacil J, D'Esposito M (2014) Distributed and dynamic storage of working memory stimulus information in extrastriate cortex. J Cogn Neurosci 26:1141-1153.

Thomas JM, Huber E, Stecker GC, Boynton GM, Saenz M, Fine I (2015) Population receptive field estimates of human auditory cortex. Neuroimage 105:428-439.

van Bergen RS, Ma WJ, Pratte MS, Jehee JF (2015) Sensory uncertainty decoded from visual cortex predicts behavior. Nat Neurosci 18:17281730.

van Kerkoerle T, Self MW, Roelfsema PR (2017) Layer-specificity in the effects of attention and working memory on activity in primary visual cortex. Nat Commun 8:13804.

White BJ, Berg DJ, Kan JY, Marino RA, Itti L, Munoz DP (2017a) Superior colliculus neurons encode a visual saliency map during free viewing of natural dynamic video. Nat Commun 8:14263.

White BJ, Kan JY, Levy R, Itti L, Munoz DP (2017b) Superior colliculus encodes visual saliency before the primary visual cortex. Proc Natl Acad Sci USA 114:9451-9456.

Winawer J, Horiguchi H, Sayres RA, Amano K, Wandell BA (2010) Mapping $\mathrm{hV} 4$ and ventral occipital cortex: the venous eclipse. J Vis 10:1.

Wurtz RH, Albano JE (1980) Visual-motor function of the primate superior colliculus. Annu Rev Neurosci 3:189-226.

Zénon A, Krauzlis RJ (2012) Attention deficits without cortical neuronal deficits. Nature 489:434-437.

Zhang P, Zhou H, Wen W, He S (2015) Layer-specific response properties of the human lateral geniculate nucleus and superior colliculus. Neuroimage 111:159-166. 